\title{
Operation Quality Indicators for Shovel-Truck Systems at Open-Pit Coal Mines
}

\author{
Anton Voronov ${ }^{1}$, Yuri Voronov ${ }^{2}$, Artyom Voronov ${ }^{2}$, and Nuray Demirel ${ }^{3}$ \\ ${ }^{1}$ JSC Kuzbassrazrezugol, 650054, Kemerovo, Russia \\ ${ }^{2}$ T.F. Gorbachev Kuzbass State Technical University, 650000, Kemerovo, Russia \\ ${ }^{3}$ Middle East Technical University, Üniversiteler Mah., Dumlupınar Bulvarı, No: 1, 06800 Çankaya, \\ Ankara, Turkey
}

\begin{abstract}
Stripping and mining operations at open-pit coal mines are performed mainly by heavy shovel-truck systems (STS). One of the main problems of the STS is a rather low level of its operation quality, an objective assessment of which is an important step in identifying the causes of low quality and effective ways to improve it. The purpose of assessing the STS operation quality is defined as a functional criterion. The next important step of the assessment is to choose the set of indicators that most characterize the STS operation quality. In this article we present the rationale, the general principles for the formation of quality indicators set, the sources and the main dependencies for its determination. For this purpose, modern methods of data collection and processing, analysis and synthesis are used. The ability to assess the STS operation quality is very important for identifying the main directions of improving its operational performance, reaching the optimization of the key performance indicators by the quality criterion, and, as a result, for the possible saving of material and technical resources in the open-pit mining of minerals.
\end{abstract}

\section{Introduction}

After establishing the functional criterion for the STS, the next most important task of assessing its operation quality is to substantiate and determine the set of indicators characterizing the STS operation quality. The basic principles of the set formation are: indicators that were used in determining the functional criterion cannot be re-used as single indicators; selected indicators should be representative, independent of each other, homogeneous (with a decrease in the indicator values, the quality should definitely improve).

\section{Materials and methods}

The set of indicators that most characterize the STS operation quality is established, first of all, depending on the selected criterion. To assess the STS operation quality, we selected the functional criterion in the following form [1]:

$$
\lambda=\frac{N_{t}}{\frac{N_{S}}{t_{L}^{S}} \cdot t_{h} \cdot k_{S T S}}
$$


which, although it is less general than economic criteria, makes it possible to assess the STS efficiency fairly objectively.

In the dependency (1) $N_{s}$ and $N_{t}$ - numbers of shovels and trucks in the STS, respectively; $t_{L}^{S}$ - time to fully load a truck by a shovel, min; $t_{h}$ - truck haul time, min; $k_{S T S}-$ coefficient of the STS operational performance.

STS is a complex dynamic system, the operation quality of which depends on a number of specific conditions: configuration of the open-pit mine, type of the material transported (overburden or mineral), structure of loading and transport equipment fleets and its condition, organization of fleet operation. World practice shows that, in recent years, the average performance of mining equipment has a strong downward trend, mainly due to increased downtime during the operation (for example, the performance of mining trucks decreased by $41 \%$ in $2006-2010$, despite new technological achievements [2,3]).

The overall STS performance is largely determined by the time to load the mining trucks and the characteristics of its movement. In addition, the number and structure of mining shovels and trucks are two of the most important factors in determining the rational parameters of open-pit mining (OPM) and the STS operation quality. If the number of simultaneously operating shovels and trucks is not balanced, there are downtimes of either trucks or shovels waiting for work. However, even if the number of shovels and trucks in the STS is balanced, downtime can occur due to the heterogeneity of shovel and truck fleets, mismatch between the trucks and the shovels serving them, untimely arrival of trucks to shovels for loading caused by irrational truck allocation between loading points.

When choosing indicators it is necessary to keep in mind the following principles of qualimetry:

- indicators that were used in determining the functional criterion can't be re-used as single indicators;

- selected indicators should be representative, which will ensure the reliability of the results obtained. As stated by one of the statements of systems engineering, a representative criterion (parameter, indicator) characterizes the implementation of a simple and clear rule: the best (worst) system uniquely corresponds to a larger criterion value;

- the set should not include indicators that are consensual, that is, they directly define each other, because the consensual parameters bring the same information;

- for indicators, with a decrease in the values of which the quality gets worse, its inverse values should be taken as single indicators.

\section{Results and discussion}

One of the most important indicators characterizing the structure of loading and transport equipment fleets is the ratio of the number of trucks and shovels in the STS (the ratio of the STS structure):

$$
k_{N}^{\prime}=\frac{N_{t}}{N_{s}} .
$$

This indicator reflects the number of trucks servicing each shovel. If trucks are not enough, then shovels idle waiting for work; if trucks are too many, then trucks idle. However, the $k_{N}^{\prime}$ indicator is directly included in the functional criterion (1), therefore, it cannot be used again, in the form of a single quality indicator.

Also, the functional criterion formula (1) includes the time to fully load a truck by a shovel $t_{L}^{S}$, the coefficient of operational performance $k_{S T S}$ and the truck haul (cycle) time $t_{h}$, which, in their pure form, cannot be included in the single indicators of the STS operation quality either. But their separate components can be the subject of further analysis. 
First of all, it is necessary to analyze the operating cycle of a mining truck for various combinations of machine sizes in the STS.

Truck cycle time can be defined as the sum of the durations of all technological operations that make up the cycle: loading $\left(t_{L_{i j}}^{S}\right)$, transporting material to the dumping point $\left(t_{L M_{i}}\right)$, dumping $\left(t_{d_{i}}\right)$, movement of an empty truck to the next loading point $\left(t_{E M_{i}}\right)$, truck downtime waiting for loading $\left(t_{D_{i j}}^{t}\right)$, maneuvering time before loading $\left(t_{m . l}\right)$ and dumping $\left(t_{m . d}\right)$. At the same time, the truck dumping time and its maneuvering time before loading and dumping are normalized (for example, by the "Instructions for the recording of working hours of technological vehicles" operating in the Kuzbassrazrezugol Company), so it makes no sense to include them in consideration.

Thus, $t_{L_{i j}}^{S}, t_{L M_{i}}, t_{E M_{i}}$ and $t_{D_{i j}}^{t}$ remain the most significant components of the truck cycle time.

The time spent by the shovel $j$ to fully load the body of the truck $i\left(t_{L_{i j}}^{S}\right)$ can be defined as the product of the duration of one operating cycle of the shovel $j\left(t_{c_{j}}^{S}\right)$ by the number of buckets of the shovel $j$ loaded into the truck $i$ for its full load $\left(n_{b_{i j}}\right)$.

Loading time in absolute form $\left(t_{L}^{S}\right)$ is included in the functional criterion and, as already mentioned above, cannot be used as a single indicator. But the components of this value, the shovel cycle time $t_{c}^{S}$ and the number of loaded buckets $n_{b}$ should be the subject of further consideration.

The cycle time for the shovel is a fixed value, but in the form of a performance indicator $E_{b}^{S} / t_{c}^{S}$ can serve as a characteristic of the STS shovel fleet. Thus, the performance indicator of the STS shovel fleet can be named the first indicator characterizing the STS operation quality:

$$
P^{s}=\frac{\widetilde{E_{b}^{s}}}{\widetilde{t_{c}^{s}}}
$$

where $\widetilde{E_{b}^{s}}, \widetilde{t_{c}^{s}}-$ weighted average bucket capacity and shovel cycle for the mine shovel fleet, respectively.

A similar indicator characterizing the truck fleet is the performance indicator of the motor vehicle fleet $P^{t}$, which can be taken as the second indicator characterizing the STS operation quality:

$$
P^{t}=\frac{\widetilde{E_{b}^{t}}}{\widetilde{t_{h}}}
$$

where $\widetilde{E_{b}^{t}}, \widetilde{t_{h}}-$ weighted average body capacity and truck haul time, respectively.

The use of weighted averages here and further is due to the fact that shovel and truck fleets at all Kuzbassrazrezugol mines are heterogeneous (mixed), but the mine should be assessed as a whole.

We weight the parameters included in dependencies (2) - (3) by the number of hauls made by trucks of certain sizes (types) from the corresponding shovels. Then we have:

$$
\widetilde{E_{b}^{s}}=\frac{\sum_{1}^{n_{j}} E_{b_{j}}^{s} \cdot n_{h_{j}}}{\sum_{1}^{n_{j}} n_{h_{j}}} ; \quad \widetilde{t_{c}^{s}}=\frac{\sum_{1}^{n_{j}} t_{c_{j}}^{s} \cdot n_{h_{j}}}{\sum_{1}^{n_{j}} n_{h_{j}}}
$$

for the shovel fleet, and 


$$
\widetilde{E_{b}^{t}}=\frac{\sum_{1}^{n_{i}} E_{b_{i}}^{t} n_{h}}{\sum_{1}^{n_{i}} n_{h_{i}}} ; \quad \widetilde{t_{h}}=\frac{\sum_{1}^{n_{j}} t_{h} \cdot n_{h_{j}}}{\sum_{1}^{n_{j}} n_{h_{j}}}
$$

for the truck fleet of the STS.

In formulas (4) and (5) $E_{b_{j}}^{S}$ - bucket capacity of a shovel of the type $j, \mathrm{~m}^{3} ; t_{c_{j}}^{S}$ - cycle time of a shovel of the type $j$, min; $n_{h_{j}}$ - number of hauls made by trucks from a shovel of the type $j ; n_{j}$ - number of shovel types in the STS; $E_{b_{i}}^{t}$ - body capacity of a truck of the type $i, \mathrm{~m}^{3} ; t_{h}$ - truck haul time from a shovel of the type $j$, min; $n_{h_{i}}$ - number of hauls made by trucks of the type $i$.

The number of shovel buckets loaded into the body of a truck for full loading $\left(n_{b}\right)$ can be the indicator characterizing the shovel-truck matching.

The weighted average number of buckets loaded into the weighted average truck by the weighted average shovel will be defined as:

$$
n_{b}=\frac{\widetilde{E_{b}^{t}}}{{\widetilde{E_{b}^{s}}}^{s}}
$$

The number of buckets in the form of (6), however, can't be an indicator of the STS operation quality, since it has limitations in value. It is known that the rational number of buckets loaded into the truck body, with optimal transportation distances of up to $5 \mathrm{~km}$, is 3-5. If the distance exceeds $5 \mathrm{~km}$, the rational number of buckets may be more than 5 . But the lower limit of 3 buckets suggests that a full body load of only 1-2 buckets leads to heavy dynamic (impact) loads on the truck chassis, which are dangerous because of a possible disruption (breakdown) of a truck and its failure.

With such restrictions, it is recommended to accept not the absolute value of the parameter (6) as a single indicator, but the difference between it and the value limiting the parameter change, based on the principles of qualimetry. On this basis, the following indicator is taken as the third indicator:

$$
n_{b}^{\prime}=n_{b}-3
$$

The most important operational indicator of the truck operation, determining the nature of movement on separate route sections in the "loaded" $\left(t_{L M_{i}}\right)$ and "empty" $\left(t_{E M_{i}}\right)$ directions, as well as the full time of a truck haul, is the speed of movement.

The technical speed of a truck $(\vartheta)$ depends not only on its structural features, but also on the operating conditions: slope of the road, complexity of the route, proportion of permanent and temporary roads, quality of the road surface, traffic intensity. The truck wear and the driver qualification also have a significant impact on speed.

The operational speed covers all the factors of technical speed and additionally takes into account the downtime of trucks during a work shift. It is defined as the total distance of each truck per shift, divided by its duration $T_{s h}(\mathrm{~km} / \mathrm{h})$ :

$$
\vartheta_{e}=\frac{2 L_{t r} \cdot n_{h}}{N_{t} \cdot T_{s h}}
$$

where $L_{t r}$ - weighted average distance of material transportation by the weighted average truck, $\mathrm{km} ; n_{h}$ - number of hauls performed by all trucks during the work shift.

Since in this case we are especially interested in downtimes, we take the operational speed, weighted average for the whole truck fleet of this mine $\left(\vartheta_{e}\right)$, as the fourth indicator characterizing the STS operation quality.

Since the downtime of loading and transport equipment (shovels and trucks), according to the functional criterion (1), has a significant impact on the level of the open-pit STS op- 
eration quality through the coefficient of the STS operational performance $\left(k_{S T S}\right)$, then all indicators related to the equipment downtime for various reasons, should be taken into account in the STS operation quality indicators.

All equipment downtimes in the STS are divided into planned and unplanned ones. Planned downtime is the downtime, the duration of which is regulated by regulatory documents for the specific conditions of mining equipment operation. They are revised only when mining conditions change. Unplanned downtime is the downtime of machines that have already been commissioned for a specific shift, but for reasons beyond the control of a shovel operator (or a truck driver) they can't work and, therefore, can't perform a shift task. These are the downtimes that can be avoided and occur solely due to the unsatisfactory condition of shovel and truck fleets and poor organization of its work. According to openpit coal mines in Kuzbass, downtime in anticipation of loading is $30-50 \%$ of all truck downtime during a shift. If we take into account the downtime associated with breakdowns and repair of shovels and trucks, then the total downtime almost doubles.

Due to the essentiality of loading and transport equipment downtime at Kuzbass openpit coal mines, an important indicator of the STS operation quality is the proportion of total shovel downtime $\left(\sum_{1}^{N_{S}} t_{D}^{S}\right)$ and truck downtime (including waiting time) $\left[\sum_{1}^{N_{t}}\left(t_{D}^{t}+t_{D}^{t . w}\right)\right]$ in the total duration of all hauls in the working shift per one truck (or the actual shift duration, taking into account all interruptions in the work $-T_{s h}$ ):

$$
k_{D}^{\Sigma}=\frac{\sum_{1}^{N_{s}} t_{D}^{s}+\sum_{1}^{N_{t}}\left(t_{D}^{t}+t_{D}^{t \cdot w}\right)}{T_{s h}} .
$$

The share of equipment downtime during the work shift $\left(k_{D}^{\Sigma}\right)$ will be the fifth indicator characterizing the STS operation.

The ratio of shovel and truck downtimes is equally important. Usually, mines aim to minimize the waiting time of shovels, even at the expense of commissioning additional trucks, which increases the waiting time of trucks. This is due to the significantly higher cost of downtime of more expensive shovels compared to the downtime of trucks. At the Kedrovsky mine, the cost of 1 hour of downtime of P\&H-2800 shovels is 136,7 thousand RUR); for EKG-15 it is 70,8 thousand RUR, for EKG-12us - 53,3 thousand RUR and for BelAZ-75306 trucks - 17,5 thousand RUR. That is, the cost of downtime of domestic medium-powered shovels is 3-4 times higher than for BelAZ trucks, and for high-powered foreign shovels it is 8-10 times higher than for BelAZ trucks.

However, despite the reduction in waiting time of shovels, its downtime for other reasons still remains high. Given the importance of this issue, we introduce the sixth indicator in the set of STS operation indicators, which reflects the ratio of downtime of shovel and truck fleets:

$$
k_{D}^{s-t}=\frac{\sum_{1}^{N s} t_{D}^{s}}{\sum_{1}^{N_{t}}\left(t_{D}^{t}+t_{D}^{t \cdot w}\right)}
$$

Downtimes characterize the unproductive use of equipment and should be minimized. Studying the experience of open-pit mine operation, which is reflected in the recent periodical scientific literature, revealed the absence of significant achievements in the field of production organization aimed at reducing equipment downtime. Moreover, leading experts in this field point out that the same methods of OPM organization are still used at Russian open-pit mines as before 50-60 years ago. As a result, the level of shovel use (including foreign high-productive shovels) did not change at most mines and remains within 50-60\% of the calendar time. It is argued that, in this matter, practice lags significantly behind the theory of the production and management organization. 
Open-pit coal mines in Kuzbass traditionally use the "fixed assignment" method of organizing the loading and transport process, when each truck is assigned to a specific shovel and dumping point at the beginning of the shift, and works on the same route during the entire shift. Trucks are re-assigned only if the operating conditions change (for example, if a shovel breaks down). Optimization, in this case, means determining the optimal number of trucks for a given shovel [4-7]. Such a system can be effective only when shovels work continuously, and trucks arrive for loading evenly. However, the equipment cannot work according to a fixed algorithm. Unpredictable and subjective factors often interfere with its work: poor rock crushing during drilling and blasting, mismatch between shovels and trucks, different quality of different road sections, impossibility of overtaking slow-moving trucks by more fast ones, varying degrees of truck wear and driver qualification, unpredictable breakdowns, etc. [8-12].

In accordance with the above, two more indicators, reflecting the ratio of working hours and the downtime of shovel and truck fleets during the shift, should be included in the set:

$$
\begin{gathered}
k_{r}^{s}=\frac{t_{L}^{s}}{t_{D}^{s}} \\
k_{r}^{t}=\frac{t_{h}-t_{D}^{t . w}}{t_{D}^{t}+t_{D}^{t . w}}
\end{gathered}
$$

where $t_{L}^{S}$ - time to load a truck by a shovel (shovel work), $\min ; t_{D}^{S}$ - downtime of shovels, $\min$; $t_{D}^{t}$ - downtime of trucks excluding waiting for loading $t_{D}^{t . w}$, min; $t_{h}$ - duration of truck hauls including waiting for loading $t_{D}^{t . w}$, min. All time parameters in formulas (8) and (9) are taken per 1 haul.

Coefficients $k_{r}^{s}$ and $k_{r}^{t}$ are, therefore, the seventh and eighth indicators of the STS operation quality.

All considered indicators are divided into four groups:

- indicators of the composition, structure and matching of loading and transport equipment fleets $\left(k_{N}^{\prime}, n_{b}^{\prime}\right)$;

- indicators of the fleet performance $\left(P^{s}, P^{t}\right.$ are for potential performance, $\vartheta_{e}$ is for actual one);

- indicators of the downtime value and structure $\left(k_{D}^{\Sigma}\right.$ and $\left.k_{D}^{S-t}\right)$;

- indicators of the productive use of fleets during the shift $\left(k_{r}^{s}\right.$ and $\left.k_{r}^{t}\right)$.

It is known from qualimetry that the most important requirement for single quality indicators is their independence. Checking the selected indicators for interconnection showed that only two indicators are dependent on each other - the indicator of the shovel fleet performance $P^{s}$ (2) and the indicator of fleet matching $n_{b}^{\prime}$ (7). The relationship between them can be explained by the fact that all the values in the formulas are fixed in value parameters of shovels and trucks, included in its technical characteristics, and don't depend on operational factors. The relationship between $n_{b}^{\prime}$ and $P^{s}$ can be obtained by statistically processing the corresponding data of open-pit coal mines. It is enough to choose one of the two interdependent indicators $\left(n_{b}^{\prime}\right.$ and $\left.P^{s}\right)$ to assess the STS operation quality. We keep the $P^{s}$ indicator.

Thus, out of eight indicators selected for assessing the STS operation quality, seven indicators remain after checking for consistency (interdependence), namely:

- performance indicator of the STS shovel fleet, $P^{s}, \mathrm{~m}^{3} / \mathrm{min}$. Since the quality improves with an increase of the indicator, then, in accordance with the principles of qualimetry, the first single indicator should be the inverse value $-1 / P^{s}$;

- performance indicator of the STS truck fleet, $P^{t}, \mathrm{~m}^{3} / \mathrm{min}$. For the same reason, we take the inverse value as the second single indicator $-1 / P^{t}$; 
- operational speed of trucks in the STS, $\vartheta_{e}, \mathrm{~km} / \mathrm{h}$. The inverse value $\left(1 / \vartheta_{e}\right)$ is taken as the third single indicator;

- share of equipment downtime in the work shift duration, $k_{D}^{\Sigma}$. As the quality improves with a decrease of the indicator, the absolute value should be taken as the fourth single indicator $-k_{D}^{\Sigma}$

- ratio of shovel and truck downtimes, $k_{D}^{S-t}$. For the same reason, the absolute value $\left(k_{D T}^{S-t}\right)$ is taken as the fifth single indicator;

- level of productive use of the STS shovel fleet, $k_{r}^{s}$. Since the quality improves with an increase of the indicator, the inverse value $\left(1 / k_{r}^{S}\right)$ should be taken as the sixth single indicator;

- level of productive use of the STS truck fleet, $k_{r}^{t}$. By analogy with the previous one, we take the inverse value $\left(1 / k_{r}^{t}\right)$ as the seventh single indicator.

\section{Conclusion}

After determining the functional criterion for the STS, the next most important task of assessing its operation quality is to substantiate and determine the set of indicators characterizing the STS operation quality. The analysis showed that to assess the STS operation quality, we can single out seven indicators, which are divided into four groups: indicators of the composition, structure and match of the fleets of loading and transport equipment (shovels and trucks); indicators of the equipment fleet performance (potential or actual); indicators of the downtime value and structure; indicators of the productive use of equipment fleets during a shift.

\section{References}

1. Y. Voronov, A. Voronov, E3S Web Conf., 21 (2017)

2. R. A. Carter, Eng. Min. J., 213:3, 28 (2012)

3. G. Lumley, Trends in performance of open cut mining equipment (GBI Mining Intelligence, 2012)

4. N. Cetin, Open-pit truck/shovel haulage system simulation (Middle East Technical University, Turkey, 2004)

5. C. Ta, J. Kresta, J. Forbes, H. Marquez, Int. J. Surf. Min. Reclam. Env., 19:3, 162 (2005)

6. A. Krause, C. Musingwini, J. S. Afr. I. Min. Metall., 107, 469 (2007)

7. S. Ercelebi, A. Bascetin, J. S. Afr. I. Min. Metall., 109, 433 (2009)

8. M. M. Vemba, J. S. Afr. I. Min. Metall., 104, 139 (2004)

9. C. Burt, An optimisation approach to materials handling in surface mines (Curtin University of Technology, Curtin, 2008)

10. C. Burt, L. Caccetta, P. Welgama, L. Fouche, J. Oper. Res. Soc., 62:8, 1498 (2011)

11. J. Krzyzanowska, J. S. Afr. I. Min. Metall., 107, 215 (2007)

12. S. V. Mkhatshwa, J. S. Afr. I. Min. Metall., 109, 223 (2009) 